\title{
Machine Learning Based Resourceful Clustering With Load Optimization for Wireless Sensor Networks
}

\author{
Dr. Jennifer S. Raj \\ Professor, ECE,Gnanamani college of Technology \\ Namakkal, India. \\ Email id: jennifer.raj@gmail.com
}

\begin{abstract}
The sensors grouped to gather to form the network of their own, in the wireless medium and communicating to the each other over radio, faces issues that leads to failure in continuous communication, causing miss communication as it is powered by batteries with limited energy availability So it becomes essential to device a perfect routing scheme that is energy efficient. Though the clustering approach was found to be highly efficient to manage the transmission from source to the target. The elected head in each cluster has to take the entire load on it as it has to gather all the data and transmit it to the base station. So it was necessary to balance the load in the network formed using the sensor and communicating in wireless medium. The GWO (Grey Wolf Optimization)-PSO (Particle Swarm Optimization) based clustering is followed in the paper to have a perfect clustering with balanced load as well as energy efficient optimization. The method followed in the paper was simulated using the network simulator-Two to identify the performance improvements in the sensor networks communicating in wireless medium.
\end{abstract}

Keywords: Sensor-Networks, Wireless Communication Medium, Balanced Load, Energy Optimization, Machine Learning

\section{Introduction}

Nowadays the networks formulated using sensors, that communicate over radio are becoming more prominent as the almost employed in a wider range of applications and are capable of being employed even in regions were human access is impossible. Theses sensor are tiny equipment's, encompassed of units such as the "sensors, micro controllers, transceivers and a battery to empower the sensing, processing and further conveying. The effective utilization of power is essential in the sensor networks as the replacing or recharging of the batteries are highly tedious. So it becomes necessary to devise and energy optimized routing to minimize the energy consumption in transferring the information's from the source data point to its aimed destination, without any interruptions or failure in communication that may be caused by the exhausted sensors. 
Journal of Ubiquitous Computing and Communication Technologies (UCCT) (2020)

Vol.02/ No. 01 Pages: 29-38

https://www.irojournals.com/jucct/

DOI: https://doi.org/10.36548/jucct.2020.1.004

Though multitude of methods were put forth, the clustering method became more popular as it optimized the energy usage to a certain extent. The clustering methodology, formed clusters utilizing the sensors holding similar capabilities and strengths. Further elects one of the sensor with superior attributes as the head to the cluster and all the other sensor in the cluster are termed as members.

Whenever the source tries to send information to a particular destination, the information from the source is transmitted to its corresponding cluster head and then to the base station, so the elected head has to hold all the information that are to be transmitted as well as route it to the proper destination, so this cause necessity for the balancing the load in the sensor networks that communicate without wires. The clustering method followed in the paper replaces the gateways for the elected head i.e. (CH's) and tries to balance the loads in the network a by engaging the "Grey Wolf Optimization and the Particle Swarm Optimization" clubbed to gather to attain an "solution using a clustering to balance the overall load of the network".

The effective solutions put forth to balance the network's load is formulated "with the related works in section 2, the preliminaries in the section 3 , the proposed work in section 4 , results evaluation in section 5 and conclusion in section 6".

\section{Related Works}

To start with the discussions on the basic of the wireless networks the related works has gone the work of the author Vieira et al [1] who elaborates the particulars of the WSN as a survey, further in the year 2004 Ulema, et al [2] discusses the protocols, structure and the management strategies followed in the sensor networks communicating in wireless medium, Xiangning et al [3] has performed the "Improvement on LEACH protocol of wireless sensor network." Alzaid et al [4] has conducted the "wireless sensor networks test-bed for the wormhole attack."

Smys, S., et al [5] proposes the "K-connection Maintenance algorithm for Balanced Routing in Mobile Ad Hoc Networks." Thushara, K.,et al [6] performs the "Dynamic Clustering and Prioritization in Vehicular Ad-hoc Networks: Zone Based approach." Sharnya, P et al [7] devises the "Self organizing wireless mesh network." An "Energy-Aware Security Routing Protocol for Wsn In Big-Data Applications." Was formulated by the author Smys, S et al [8] in the year 2019.

"Ramesh, S et al [9] in the year 2017 devised a clustering architecture, that is heuristic and software based to enhance the performance of the Mobile adhoc network". Jennifer S. Raj.et al [10] introduced the authentication scheme in the WANET to have a performance optimization, and Duraipandian, M at al [11]

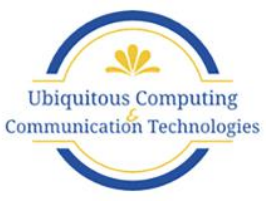


utilized the machine learning strategies to improve the mobile adhoc networks performance. A. Anto Prem Kumar et al [12] devised efficient routing with proper localization for the sensor networks that are formed as clusters. Raj, Jennifer S et al [13] conducted the "QoS optimization of energy efficient routing in IoT wireless sensor networks"

\section{Optimization Techniques Used}

\subsection{Grey-Wolf Optimization}

The Grey Wolf Optimizer was devised by Mirjalili et al [14] Inspired by the hierarchy of leadership and the conduct of hunting in the grey-wolves. The algorithm begins with the natural initialization of the wolves' population in a random manner, aiming to attain the optimal three solutions in the first three positions $\left(O P_{1}, O P_{2}, O P_{3}\right)$ the vector form representation of the wolves' is given s shown in equation 1

$$
\vec{A}=\overrightarrow{a_{1}}+\overrightarrow{a_{2}}+\cdots \overrightarrow{a_{n}}
$$

With $\mathrm{n}=$ search space dimension and the three optimal solutions move towards the location of the target and the rest of the pack $\left(R_{P}\right)$ tracks their path, the three fundamental stages of wolves involved in the hunting strategy of the wolves" were "encircling, attacking and hunting" the encircling of the prey is mathematically calculated, estimating the distance by subtracting the location of the prey and the wolves, and multiplying it with the coefficient vectors. The hunting is performed after the location of the food is well studied by the three optimal solutions and the $R_{P}$ is updates its positions according to the optimal solutions. In this the distance for each optimal solution is estimated by subtracting the location of the corresponding optimal solution with the $R_{P}$ in the attacking processing the "grey wolves diverge from each other while searching for prey (exploration) and converge while attacking the prey (Exploitation)". The equations below are framed in this regard.

Distance calculation on encircling stage is represented as:

$$
\overrightarrow{d l s t}=\left|\vec{c} \cdot \overrightarrow{\text { prey }}_{\text {position }}(t)-\overrightarrow{\text { wolf }}_{\text {position }}(t)\right| \text { Where } \mathrm{C} \text { is a coefficient Vector }
$$

Distance for each optimal solution on hunting stage is given as

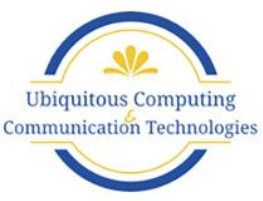


Journal of Ubiquitous Computing and Communication Technologies (UCCT) (2020)

Vol.02/ No. 01 Pages: 29-38

https://www.irojournals.com/jucct/

DOI: https://doi.org/10.36548/jucct.2020.1.004

$$
\begin{aligned}
& \overrightarrow{d \iota s t}_{O P 1}=\left|\vec{c}_{1} \cdot \overrightarrow{\text { positlon }}_{o p 1}-\overrightarrow{\text { wolf }}_{\text {position }}\right| \\
& \overrightarrow{d \iota s t}_{O P 2}=\left|\vec{c}_{2} \cdot \overrightarrow{\text { positlon }}_{o p 2}-\overrightarrow{\text { wolf }}_{\text {position }}\right| \\
& \overrightarrow{d \iota s t}_{O P 3}=\left|\vec{c}_{3} \cdot \overrightarrow{\text { positlon }}_{o p 3}-\overrightarrow{\text { wolf }}_{\text {position }}\right|
\end{aligned}
$$

\subsection{Particle Swarm Optimization [15]}

The foraging behavior of the swarm was utilized by the author to devise the particle swarm optimization, "It is a population based stochastic optimization technique developed by Dr.Eberhart and Dr.Kennedy in 1995 , inspired by the social behavior of the bird flocking"

It also begins with the initialization of population and sorts out for the optimal by updating the generations. The potential solutions of the PSO are particles. The activities of the bird flocking are simulated in PSO, every individual answers in the PSO is termed as the particle (bird) "All particles have fitness values that are assessed to be optimized by the fitness function and velocities that guide particle flight. By following the current optimum particles the particles fly through the space of the question". It is usually initiated with a group of "random particles" and sorts out for the optimal one by updating the position and the velocity of the each particle in each iteration. The fitness and the tracking capability of each particle in the population is estimated as the Pbest and Gbest in PSO the velocity and the position update are obtained as shown in equation. (6) and (7)

$$
\begin{aligned}
& \text { Velocity }(t+1)=\text { velocity }(t)+C 1 . \text { Rand }(t) \cdot(\operatorname{Pbest}(t)-\text { position }(t))+ \\
& \text { C2 Rand }(t) .(\operatorname{Gbest}(t)-\text { positon }(t))
\end{aligned}
$$

$$
\text { Position }(t+1)=\text { Position }(t)+\text { velocity }(t)
$$

"The pseudo code for the PSO is as follows" in figure .1 
Journal of Ubiquitous Computing and Communication Technologies (UCCT) (2020)

Vol.02/ No. 01 Pages: 29-38

https://www.irojournals.com/jucct/

DOI: https://doi.org/10.36548/jucct.2020.1.004

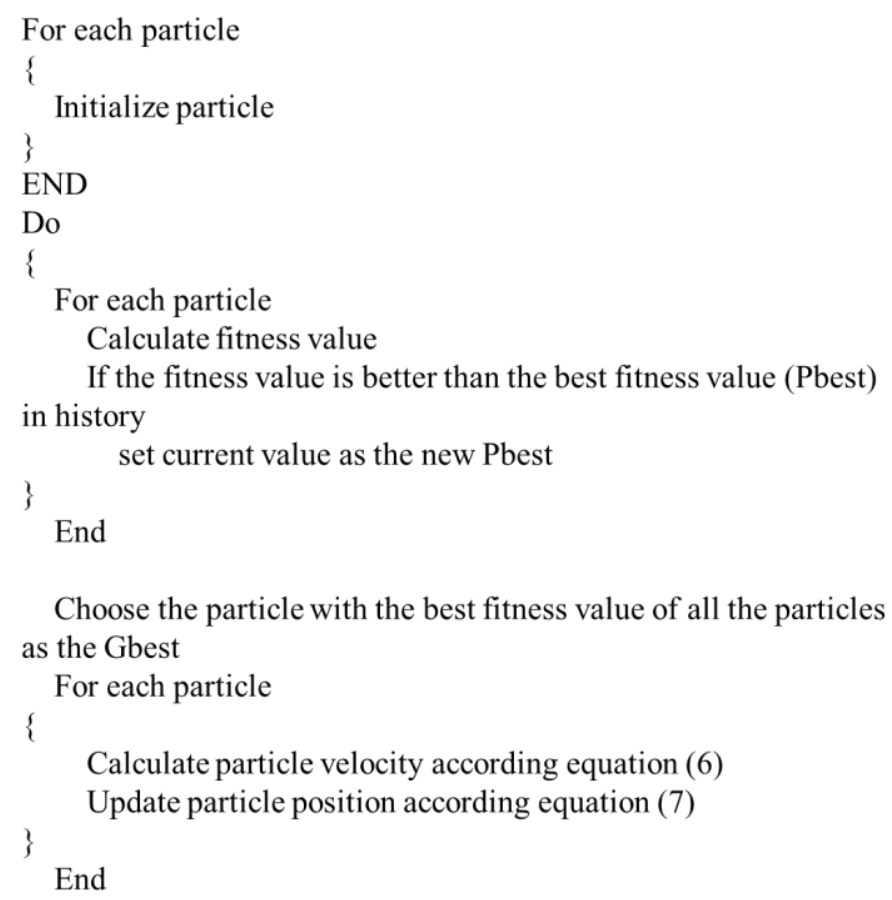

Figure.1 Pseudo Code of PSO

\subsection{Proposed Methodology}

The application of the GWO -PSO for attaining a clustering with balanced load for the sensor networks that are communicating in the wireless medium initiates with the approach of grey wolf optimization and starts the first phase of initializing the population, the population involves the gateways and the sensors initialization. "The next phase evaluates the fitness $\left(\right.$ Gate $\left._{F i t}\right)$ and determines the optimal gateways utilizing the number of gateways determined (Gate load $_{\text {lo }}$, number of gates (Gate balanced $_{\text {) }}$ ) that are balanced among the number of gateways $\left(\right.$ Gate $\left._{\text {total }}\right)$ that were initialized" as shown in equation.8

$$
\text { Gate }_{\text {Fit }}=\text { estimated }_{\text {Gate }_{\text {load }}}\left[\text { Gate }_{\text {balanced }} / \text { Gate }_{\text {total }}\right]
$$


Journal of Ubiquitous Computing and Communication Technologies (UCCT) (2020)

Vol.02/ No. 01 Pages: 29-38

https://www.irojournals.com/jucct/

DOI: https://doi.org/10.36548/jucct.2020.1.004

With the estimated $_{\text {Gate }_{\text {load }}}=$ summation of the load that is distributed/Gate $_{\text {total }}$. The number of the loads that were distributed are calculated estimating the number of loads distributed for the maximum loads, the \# of packets sent from the source is represented by the loads distributed, as shown in equation 9

$$
\text { Distriuted }_{\text {loads }}=\text { number of bits } * \text { number of packets sent }
$$

The next step segregates the gateways that are "balanced and unbalanced" by checking them with them the maximum and the minimum verge level set for the load. The balanced ones maintains a load between the maximum and minimum verges set. The equations 10,11, 12 are framed in this regard.

$$
\begin{gathered}
\text { Maximum }=(\text { Mean } * X)+\text { Mean Where ' } \mathrm{X} \text { ' is a Constant Value } \\
\text { Minumum }=\text { Mean }-(X * \text { Mean })
\end{gathered}
$$

Where the mean was determined estimating thesummation of the load that is distributed/ maximum load. "The next phase obtains the three optimal solutions that are essential for the next phase and other solutions update their positions according to the three solutions obtained. Followed by the operations of the GWO, the PSO is done, and the fitness are evaluated as in equations 9-11 and then the optimal solution are arranged in the ascending order, based on the fittest solution obtained the each data point is assigned to the optimal gateway that lies nearest to the sensors.

\section{Results Analysis}

The proposed methodology, is analyzed by experimenting on the network simulator-2, employing hundred to one hundred and two hundred of sensor engaged with varying number of "gateways" over an area of $100 * 100 \mathrm{~m}^{2}$. The initial energy assigned for the data points and the gateways were 100 joules and 200 joules respectively. The experiment was carried out estimating the fitness of the cluster for the many methods such as GWO, GA, PSO-GA and GWO-PSO, the results observed rom each method were compared as shown in figure. 2 
Journal of Ubiquitous Computing and Communication Technologies (UCCT) (2020)

Vol.02/ No. 01 Pages: 29-38

https://www.irojournals.com/jucct/

DOI: https://doi.org/10.36548/jucct.2020.1.004

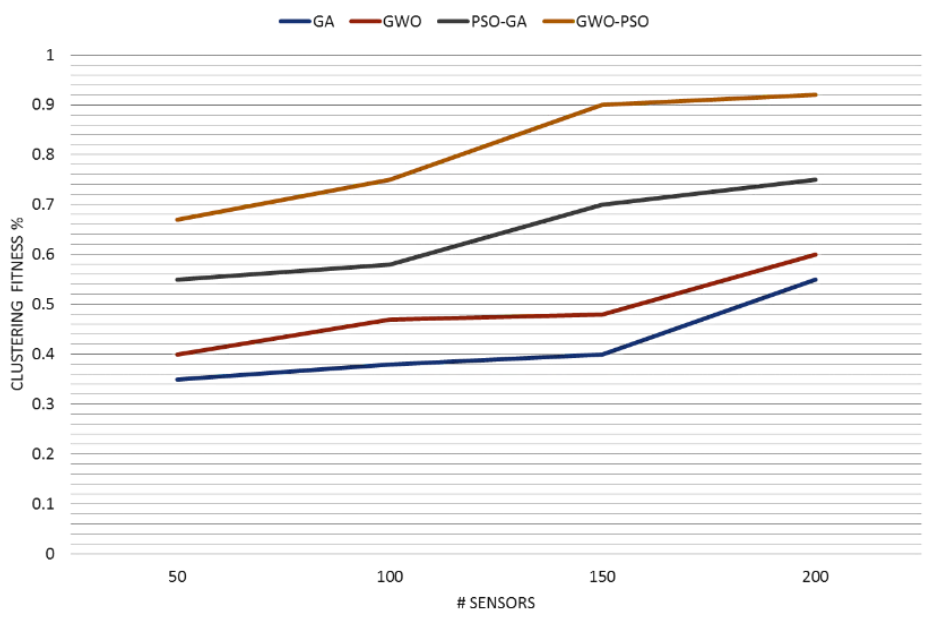

Figure.2 Clustering Fitness

From the results observed and comparison done as in figure. 2 the GWO-PSO, performed better compared to the other methods such as GWO, GA, and the PSO-GA.

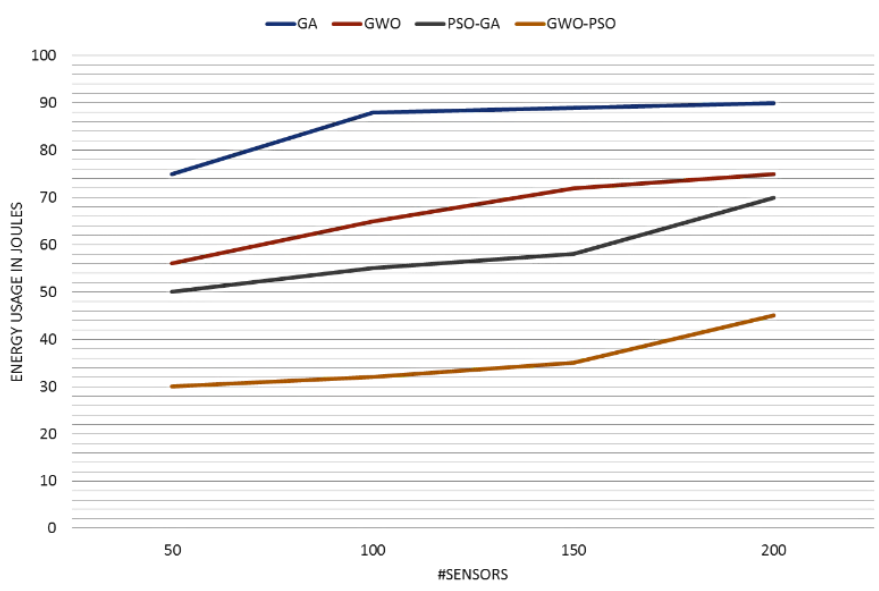

Figure.3 Energy Usage 
Journal of Ubiquitous Computing and Communication Technologies (UCCT) (2020)

Vol.02/ No. 01 Pages: 29-38

https://www.irojournals.com/jucct/

DOI: https://doi.org/10.36548/jucct.2020.1.004

The results obtained in figure. 3 show the comparison of results observed on the energy usage of the proposed method along with the other prevailing methodologies. The energy usage of the sensors in conveying the information were improved through the GWO-PSO, compared to the other methods.

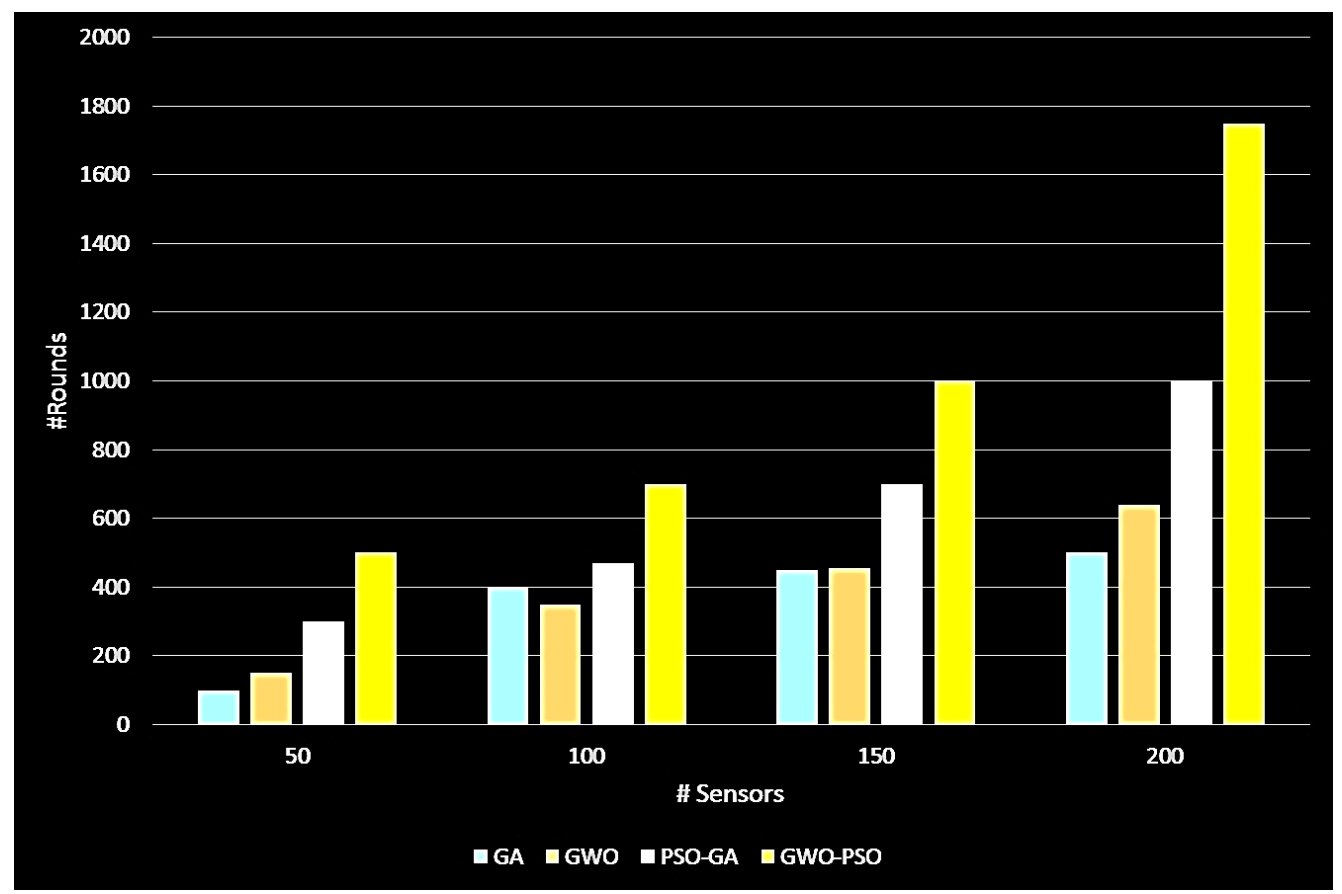

Figure.4 Energy Efficiency

The energy efficiency was checked for the GWO-PSO, based on the number of alive nodes and it was found that the very first data point gets exhausted in the GWO-PSO after completing a very higher \# of rounds, unlike the other methods whose initial node gets exhausted on completion of few rounds. They rest of the nodes were found alive in the network improving the stability of the network. So the proposed methodology utilizing the GWO-PSO performs well compared to the other methods.

\section{Conclusion}

The paper integrates the GWO with PSO to balance the load in the wireless sensor networks. The balancing of the load is initiated with the GWO followed by the PSO and the best solutions are estimated evaluating the fitness. The proposed methodology was simulated in network simulator- 2 and was evaluated as well 
Journal of Ubiquitous Computing and Communication Technologies (UCCT) (2020)

Vol.02/ No. 01 Pages: 29-38

https://www.irojournals.com/jucct/

DOI: https://doi.org/10.36548/jucct.2020.1.004

compared with three other methods and was to produce convincing results compared to the other existing methods. The GWO-PSO leaves behind the other algorithms compared on the basis of the element of network by incorporating the exclusive assets of PSO and GWO.

\section{References}

[1] Vieira, Marcos Augusto M., Claudionor N. Coelho, D. C. Da Silva, and José Monteiro da Mata. "Survey on wireless sensor network devices." In EFTA 2003. 2003 IEEE Conference on Emerging Technologies and Factory Automation. Proceedings (Cat. No. 03TH8696), vol. 1, pp. 537-544. IEEE, 2003.

[2] Ulema, Mehmet. "Wireless sensor networks: architectures, protocols, and management." In 2004 IEEE/IFIP Network Operations and Management Symposium (IEEE Cat. No. 04CH37507), vol. 1, pp. 931-Vol. IEEE, 2004.

[3] Xiangning, Fan, and Song Yulin. "Improvement on LEACH protocol of wireless sensor network." In 2007 international conference on sensor technologies and applications (SENSORCOMM 2007), pp. 260-264. IEEE, 2007.

[4] Alzaid, Hani, and Suhail Abanmi. "A wireless sensor networks test-bed for the wormhole attack." In International Journal of Digital Content Technology and its Applications. 2009.

[5] Smys, S., and G. Josemin Bala. "K-connection Maintenance algorithm for Balanced Routing in Mobile Ad Hoc Networks." International Journal of Computer Networks and Communications (IJCNC) 1, no. 3 (2009): 105-11.

[6] Thushara, K., and Jennifer S. Raj. "Dynamic Clustering and Prioritization in Vehicular Ad-hoc Networks: Zone Based approach." International Journal of Innovation and Applied Studies 3, no. 2 (2013): 535-540.

[7] Sharnya, P., and Jennifer S. Raj. "Self organizing wireless mesh network." International Journal of Innovation and Applied Studies 3, no. 2 (2013): 486-492.

[8] Smys, S. "Energy-Aware Security Routing Protocol For Wsn In Big-Data Applications." Journal of ISMAC 1, no. 01 (2019): 38-55.

[9] Ramesh, S., and S. Smys. "A software-based heuristic clustered (sbhc) architecture for the performance improvement in manet." Wireless Personal Communications 97, no. 4 (2017): 6343-6355

[10] Smys, S., and Jennifer S. Raj. "Performance Optimization of Wireless Adhoc Networks with Authentication." Journal of Ubiquitous Computing and Communication Technologies (UCCT) 1, no. 02 (2019): 64-75.

[11] Duraipandian, M. "Performance Evaluation Of Routing Algorithm For Manet Based On The Machine Learning Techniques." Journal of trends in Computer Science and Smart technology (TCSST) 1, no. 01 (2019): 25-38. 
Journal of Ubiquitous Computing and Communication Technologies (UCCT) (2020)

Vol.02/ No. 01 Pages: 29-38

https://www.irojournals.com/jucct/

DOI: https://doi.org/10.36548/jucct.2020.1.004

[12] Raj, Jennifer S., and A. Anto Prem Kumar. "Energy Efficient Localization and Routing Strategy for Cluster Based Sensor Networks."

[13] Raj, Jennifer S. "QoS optimization of energy efficient routing in IoT wireless sensor networks." Journal of ISMAC 1, no. 01 (2019): 12-23.

[14] Mirjalili, Seyedali, Shahrzad Saremi, Seyed Mohammad Mirjalili, and Leandro dos S. Coelho. "Multi-objective grey wolf optimizer: a novel algorithm for multi-criterion optimization." Expert Systems with Applications 47 (2016): 106-119.

[15] Kennedy, Jim, and Russ Eberhart. "Tutorial on particle swarm optimization." In 2002 World Congress on Computational Intelligence WCCI. 2002. 